\title{
PERFIL DE LAS FAMILIAS ESPAÑOLAS EN LAS QUE CONVIVEN TRES GENERACIONES*
}

\section{DESCRIPTIVE PROFILE OF SPANISH FAMILY UN WHICH THREE GENERATION COEXIST}

\author{
MAGDALENA SAENZ DE JUBERA OCÓN \\ Universidad de la Rioja \\ Rosa ANA ALONSO RUIZ \\ Universidad de la Rioja
}

\author{
ANA PONCE DE LEÓN ELIZONDO \\ Universidad de la Rioja \\ ÁngEl DE-JUANAS OLIVA \\ UNED
}

\section{RESUMEN}

Las familias en las que conviven tres generaciones constituyen un caldo de cultivo fundamental para la socialización y la educación en valores de sus miembros, por lo que se estima fundamental conocer y comprender en profundidad la institución familiar.

El objetivo del presente artículo se centra en perfilar algunas características de las familias españolas que están configuradas por miembros de tres generaciones, mediante el análisis de algunas variables sociodemográficas como el nivel académico, la situación profesional de los progenitores o el tipo de centro al que acuden sus hijos e hijas -

\footnotetext{
* Resultados de la investigación "De los tiempos educativos a los tiempos sociales: la cotidianidad familiar en la construcción del ocio físico-deportivo juvenil" (EDU2012-39080-C07-05) [años 20122015], y fundamento de la actual investigación "Ocio y bienestar en clave intergeneracional: de la cotidianidad familiar a la innovación social en las redes abuelos-nietos" (EDU2017-85642-R) [años 2017-2020]; ambas cofinanciadas en el marco del Plan Nacional I+D+I con cargo a dos ayudas del Ministerio de Ciencia, Innovación y Universidades, y del Fondo Europeo de Desarrollo Regional (FEDER).
} 
religioso o laico-, con el fin de establecer líneas de actuación que optimicen las relaciones intergeneracionales $\mathrm{y}$, por ende, la calidad de vida de todos y cada uno de los individuos que nutren la institución familiar. Se adopta un enfoque metodológico de carácter cuantitativo, desarrollado mediante la aplicación de un cuestionario elaborado ad hoc a una muestra de 1.764 familias de todo el territorio español. El análisis de los datos se configura en dos fases, un análisis descriptivo y un análisis inferencial bivariado, a través de tablas de contingencia. Los resultados evidencian que ni el nivel de estudios ni la situación profesional de los progenitores configuran este perfil familiar y que tan solo el tipo de familia se establece en un factor asociado, pues se descubre que cuando los progenitores están separados y sus hijos cursan estudios en centros laicos, es mayor el porcentaje de hogares trigeneracionales.

Palabras clave: familia, relaciones intergeneracionales, abuelos, nietos.

\section{ABSTRACT}

The families un which three generation coexist constituye a productive ground for the socialization and the values education of its members, reason why is not considered fundamental to know and to understand un depth the family institution.

The objective of this article is to outline some characteristics of Spanish families that are configured by members of three generations, by analyzing some sociodemographic variables such as the academic level, the professional status of parents or the type of center they choose to their sons and daughters - religious or lay - in order to desing lines of action that optimize intergenerational relationships and, therefore, the quality of life of each and every one of the individuals that nurture the family institution.

A methodological approach of a quantitative character was adopted, developed through the application of a questionnaire prepared specifically for this purpose to a sample of 1,764 families throughout the Spanish territory. The analysis of the data is configured in two phases, a descriptive analysis and a bivariate inferential analysis, through contingency tables. The results show that neither the educational level nor the professional situation of the parents configure this family profile and that only the type of family is established in an associated factor, since it is discovered that when the parents are separated and their children are studying in secular centers, the percentage of trigenerational households is higher.

Keywords: family, intergenerational relationship, grandparents, grandchildren. 


\section{INTRODUCCIÓN}

La comprensión de los orígenes de la familia pasa por entender los comienzos de nuestra especie y los sentimientos que unieron a sus componentes, por lo que como afirman algunos autores (Encinas, 2007) hablar de familia es hacer referencia a la historia de Homo sapiens.

La sociedad contemporánea se caracteriza por constantes y vertiginosos cambios que suceden en mundos reales y virtuales, de lo que deriva un escenario complejo configurado por diversidad de estructuras y tipos de familia, complicando establecer una definición válida para todas sus modalidades. Todo ello da como resultado una mayor apertura de la institución familiar a la heterogeneidad, tanto en su tamaño y composición como en las relaciones que mantiene con otras estructuras socio-institucionales (Valdemoros, Ponce de León, Sanz, y Caride, 2014).

Teniendo en cuenta las múltiples realidades y las matizaciones que conlleva, la familia puede definir como un sistema formalizado, abierto, propositivo y autorregulado, con estructuras jerárquicas entre sus miembros, que se regulan por normas que delimitan sus relaciones intra e interfamiliares, cuya finalidad es salvaguardar la pertenencia de sus miembros, quienes comparten lazos de afecto e intimidad (Álvarez y Rodríguez, 2008; García-Roca, 1999; Romero, 2002), caracterizándose las relaciones familiares por la libertad, es decir, por aceptar y amar a cada cual por ser quien es (Sellés, 2006).

En la Carta dirigida a todas las familias del mundo, con motivo del Año internacional de la familia promocionado por la Organización de Naciones Unidas en 1994, Juan Pablo II ya se refería a la familia como "comunión de generaciones". La pertenencia familiar, afectiva y efectiva se hace extensiva a los abuelos y a los nietos, premisa que ha sido constatada por diferentes estudios que señalan que para entender y aprovechar las dinámicas familiares es necesario funcionar, como mínimo, con tres generaciones (Cavalotti, 2015).

La institución familiar es la unidad primordial de convivencia y de actuación, donde las relaciones que se establecen van moldeando a los hijos e hijas que componen el hogar familiar. Se influye en su formación integral, toda vez que queda probado el papel fundamental que tiene en la transferencia de los valores, los comportamientos y las actitudes que resultan en las experiencias vitales compartidas entre sus miembros (Brazelton y Greenspan, 2005; Valdemoros et al., 2014), transfiriendo las bases de comportamientos ciudadanos para coexistir en el enfoque del bienestar personal y comunitario (Castillo, 2018). 
Badenes y López (2011) esgrimen tres razones por las que la familia es el escenario óptimo para la adquisición de los valores, para la convivencia y para la socialización en la cotidianidad (Elzo, 2004), si bien, matizan posiciones distintas si se refieren a los padres y a los abuelos. La primera se centra en el ámbito de la afectividad, donde los padres deben combinar afectos y exigencia, mientras que los abuelos experimentan relaciones emocionales más abiertas; la segunda razón estriba en la esfera de lo social, y es que los abuelos acercan a los más pequeños a una realidad a la que sus padres no llegan, como es la salud, la cultura o las formas de vida; y el último motivo se refiere a la unión entre generaciones, jugando un papel primordial los abuelos, entendiendo a la familia como red de solidaridad intergeneracional capital para su cohesión. Esta red es catalogada por la Organización Nacional de Naciones Unidas como una cuestión de interés internacional en el año 2014 e instan a agentes sociales y gubernamentales a promocionar programas intergeneracionales que promuevan el intercambio y el apoyo entre generaciones.

En los últimos años factores como el importante aumento de la longevidad o la crisis económica acontecida, han derivado en que la población mayor de 65 años se haya duplicado. También ha tenido su repercusión en la modificación de la estructura y las funciones de la familia. La institución familiar también se está transformando como reflejo de la sociedad, ya que junto a las familias nucleares tradicionales formadas por parejas casadas con hijos biológicos, tienen cada vez más presencia familias monoparentales y recompuestas, es decir, procedentes de uniones anteriores, con unas características que las alejan del modelo básico de familia (Bolívar, 2006; De-Juanas y García-Castilla, 2018), lo que provoca numerosas dificultades para su organización y para la vida en comunidad.

Las personas de avanzada edad, referidas también como "tercera edad activa" (Organización Mundial de la Salud, 2015) se erigen, con los cambios en la configuración de la familia, en piezas clave para la existencia misma de la familia actual y en protagonistas principales en la sociedad contemporánea, no tanto por la edad en la que son abuelos, que sufre mucha variabilidad, sino porque perviven en esta etapa vital durante un período de tiempo muy extenso (Peña y Pezzoli, 2018).

El estudio realizado por Sigma Dos para la Organización no gubernamental "Mensajeros de la paz", presidida por el padre Ángel García, y publicada con motivo del Día internacional de los abuelos del año 2015, así lo confirma. Según los resultados obtenidos en este estudio, que testa la opinión de las personas mayores españolas sobre temas que les afectan directamente, el 55,9\% de los 
abuelos españoles declara que ayuda a sus hijos, ya sea en el ámbito económico o en el cuidado de los nietos. De este modo, las personas mayores se convierten en un agente familiar activo en los hogares con niños que protegen y cuidan de la familia, adquiriendo una valiosa función social y educativa.

En España existe un fuerte apoyo familiar entre generaciones, tal y como lo reflejan los datos de la última encuesta europea SHARE (Survey of Health, Ageing and Retirement in Europe, Encuesta de Salud, Envejecimiento y Jubilación en Europa), que ofrecen información sobre el estado socioeconómico, la salud, las redes sociales y familiares de ciudadanos de más de 50 años procedentes de 20 países europeos, más Israel. Los resultados constatan que un 36,8\% de los abuelos españoles afirma haber cuidado de sus nietos en los últimos doce meses. Aunque el porcentaje es importante, es superado por países como Dinamarca $(55,9 \%)$, Suecia $(53,7 \%)$ y Holanda $(52,9 \%)$. Si bien, España está a la cabeza de Europa en cuanto a la intensidad de cuidado, pues un $14,1 \%$ de abuelos cuidan a sus nietos más o menos cada día, solo superado por Italia, con un $19,4 \%$. Así lo atestigua la secretaria general de The Family Watch, María José Olesti, en declaraciones a Europa Press (2016), que puso de manifiesto la labor cardinal que desempeñan los abuelos en pro de las relaciones intergeneracionales, aseverando que "son muchos los padres y madres que pueden salir a trabajar y que pueden tener un equilibro entre familia y trabajo, gracias a esa labor maravillosa que realizan muchos abuelos".

En muchas ocasiones, este cuidado se produce en la convivencia del propio hogar familiar, porque cada vez es más frecuente que coexistan varias generaciones de una misma familia, bien porque los hijos dilatan su permanencia en el hogar familiar, bien porque retornan a casa tras emanciparse y/o azotarles el desempleo. Así, desde el comienzo de la crisis esta modalidad de hogar creció sustancialmente, duplicándose entre 2007 y 2013 el porcentaje de niños menores de 16 años que conviven con personas de más de 65 años, pasando de 144.215 $(2,2 \%)$ a $333.689(4,4 \%)$, tal y como reflejan los datos de la encuesta de condiciones de vida. Es este colectivo, en muchas ocasiones, el mayor sustentador del hogar, al identificar que su porcentaje también creció durante la primera etapa de la crisis, pasando del 1,8\% en 2007 al 3\% en 2011 (Marí-Klose y Escapa, 2015).

Las bondades que ofrecen las familias multigeneracionales son enormemente relevantes pues, como afirman estudios previos (González Bernal y de la Fuente, 2007) favorecen el bienestar personal y la calidad de vida en familia, al aportar beneficios individuales y sociales asociados a la cohesión, la flexibilidad y la comunicación familiar, lo que contribuye a la creación de vínculos socioe- 
mocionales más sólidos, incrementándose la complicidad y la alianza en el ámbito familiar (Osuna, 2006).

Prestigiosos autores (Aranda, 2013; MacCallum et al., 2006; Martínez de Miguel, Escarbajal, y Moreno, 2012) enfatizan la existencia de numerosas virtudes derivadas de las relaciones intergeneracionales en el seno familiar para la salud integral de sus componentes. Así, en los mayores se desarrolla la vitalidad, optimiza los recursos para afrontar la enfermedad mental, aumenta la sensación de estima personal, fomenta la motivación, y aporta ilusión y alegría. A su vez, en los más pequeños incrementa la autoestima y la autoconfianza, evita el aisla-miento, contribuye en la prevención de hábitos nocivos, acrecienta el optimismo, aumenta la tolerancia ante la adversidad, y aporta disfrute y alegría.

En otro orden de cosas, no conviene obviar que la convivencia en una familia formada por varias generaciones también puede provocar situaciones estresantes que generan malestar. Están relacionadas con una atención excesiva u obligada a los nietos por parte de los abuelos, nuevas responsabilidades familiares o disminución de la privacidad o del espacio compartido, constituyendo realidades que obligan a velar por la calidad de las relaciones intergeneracionales en hogares multigeneracionales (Megías y Ballesteros, 2011).

Tal y como se ha avanzado, la literatura científica asevera que la familia es el principal caldo de cultivo para la socialización y la educación en valores de sus miembros (Álvarez y Rodríguez, 2008; Demaray \& Malecki, 2002; Jackson \& Warren, 2000; Juang \& Silbereisen, 1999), de lo que deriva la importancia de una coherente toma de decisiones, en pro de una educación de calidad para los hijos e hijas, así como para viabilizar un óptimo funcionamiento familiar. Por ello, para una mayor comprensión de la familia, que posibilite el establecimiento de líneas para la reflexión y la acción que favorezcan su ámbito axiológico, se estima cardinal el análisis de variables como la elección del tipo de centro educativo -religioso o laico-, el nivel académico de los progenitores, o las características del lugar de residencia familiar (Escardíbul y Villarroya, 2009; De Fuenmayor, Granell, y Villareal, 2003; Mancebón y Pérez, 2007), lo que contribuirá a la calidad de vida en familia.

Si bien son numerosos los estudios realizados sobre las familias españolas, son escasos los que se focalizan en las características de aquellas en las que conviven tres generaciones. El objetivo del presente artículo se centra en perfilar algunas características de las familias españolas que están configuradas por miembros de tres generaciones, mediante el examen de algunas variables sociodemográficas como el nivel académico de los padres y situación profesional de 
los progenitores y el tipo de centro al que acuden sus hijos e hijas, con el fin de establecer líneas de actuación que optimicen las relaciones intergeneracionales $\mathrm{y}$, por ende, la calidad de vida de todos y cada uno de los individuos que nutren la institución familiar.

\section{METODOLOGÍA}

La población de estudio de esta investigación se define por las familias residentes en España con algún estudiante matriculado en Educación Secundaria Postobligatoria.

Dadas las dificultades para conocer el tamaño de este universo se decide tomar como referencia de unidad poblacional cada estudiante de Educación Secundaria Postobligatoria. Los datos reflejados en la estadística de las Enseñanzas no universitarias de la Subdirección General de Estadística y Estudios del Ministerio de Educación, Cultura y Deporte sitúan el tamaño poblacional en 1.055.532 familias con estudiantes de Educación Secundaria Postobligatoria.

Partiendo de un universo finito tan amplio se decidió trabajar con una selección muestral representativa, para ello se optó por fijar un error de muestreo de $\pm 2,3$ sigmas, un nivel de confianza del $95 \%$ y considerar el supuesto de que $\mathrm{p}=\mathrm{q}=0,5$. Con estos criterios, el tamaño muestral ascendió a 1.764 estudiantes.

El tipo de muestreo seleccionado fue probabilístico estratificado con afijación proporcional reflejando la representatividad de las familias distribuidas por toda la geografía del estado español asegurando la proporcionalidad en las 6 zonas territoriales delimitadas por el instituto de investigación de mercados Nielsen (Tabla 1), por lo que se mantuvo una afijación ajustada en cada una de estas 6 zonas a través de un muestreo aleatorio simple.

Tabla 1: Agrupación territorial para la muestra

\begin{tabular}{ll}
\hline $\begin{array}{l}\text { Denominación/ } \\
\text { Localización }\end{array}$ & Comunidades y/o provincias \\
$\begin{array}{l}\text { A1-Noreste } \\
\text { A2-Levante }\end{array}$ & $\begin{array}{l}\text { Cataluña, Aragón (excepto Teruel) y Baleares } \\
\text { Comunidad Valenciana, Murcia y Albacete }\end{array}$ \\
A3-Sur & Andalucía, Islas Canarias, Ceuta y Melilla \\
A4-Centro & $\begin{array}{l}\text { Madrid, Castilla-La Mancha (excepto Albacete), Castilla-León } \\
\text { (excepto León, Palencia y Burgos), Cáceres y Teruel }\end{array}$ \\
$\begin{array}{l}\text { A5-Noroeste } \\
\text { A6-Norte }\end{array}$ & Galicia, Asturias y León \\
\hline
\end{tabular}

Fuente: elaboración propia 
De las 1.764 familias participantes en la muestra, el 24,3\% $(\mathrm{n}=428)$ residían en la zona centro, el 21,9\% $(\mathrm{n}=386)$ en el área sur, el 16,3\% $(\mathrm{n}=288)$ en levante, el $15,7 \%(n=277)$ en el noreste, el $9 \%(n=159)$ en la zona noroeste y el $12,3 \%$ $(n=226)$ en el norte.

Un total de siete variables articularon el análisis de este estudio que permitió definir el perfil de las familias españolas en las que conviven 3 generaciones (abuelos, padres e hijos) y que confían la educación curricular de los hijos en centros católicos. A continuación, se define cada una de ellas.

- Convivencia del abuelo en el domicilio familiar: Esta variable dicotómica, con las opciones sí/no, identifica si los abuelos viven en el domicilio habitual del estudiante de secundaria postobligatoria.

- Titularidad del centro en el que estudian los hijos: Se trata de una variable dicotómica, con las opciones católica/no católica, que identifica aquellos estudiantes que estudian educación secundaria postobligatoria en centros católicos.

- Situación familiar: Pretende identificar, a través de 5 categorías, el tipo de familia en función de la composición nuclear de la misma. Las categorías son:

- Sus padres viven juntos

- Sus padres viven separados

- Solo tiene madre o padre

- No tiene madre ni padre

- Otra

- Nivel de estudios del padre: A través de una escala ordinal de 4 puntos se mide el máximo nivel de estudios alcanzado por el padre. Las categorías contempladas son:

1: No ha finalizado ningún estudio

2: Estudios primarios (EGB)

3: Estudios secundarios (Bachillerato o FP)

4: Estudios superiores (Universidad)

- Nivel de estudios de la madre: A través de una escala ordinal de 4 puntos se mide el máximo nivel de estudios alcanzado por la madre. Las categorías contempladas son:

1: No ha finalizado ningún estudio

2: Estudios primarios (EGB)

3: Estudios secundarios (Bachillerato o FP) 
4: Estudios superiores (Universidad).

- Situación profesional del padre: Esta variable categórica registra la dedicación principal laboral del progenitor. Las categorías son:

- Empleado por cuenta ajena

- Empleado por cuenta propia

- Paro

- Jubilado

- Pensionista

- Situación profesional de la madre: Esta variable categórica registra la dedicación principal laboral de la progenitora. Las categorías son:

- Empleada por cuenta ajena

- Empleada por cuenta propia

- Paro

- Jubilada

- Pensionista

Para la recopilación de información referente a estas siete variables se aplicó el cuestionario "De los tiempos educativos a los tiempos sociales: la construcción cotidiana de la condición juvenil en una sociedad de redes. Problemáticas y alternativas pedagógico-sociales", elaborado para profundizar sobre características del alumno, vida en el centro escolar, vida familiar, tiempo libre, salud y calidad de vida, estudios y mercado laboral en el futuro, emprendimiento y funcionamiento familiar.

El instrumento fue validado a través de una prueba piloto y de un juicio de expertos. La prueba piloto se aplicó a 248 estudiantes de ocho Comunidades Autónomas y de diferentes tipos de estudios de educación secundaria postobligatoria y en el juicio de expertos participaron catorce investigadores de siete universidades españolas diferentes.

Para la aplicación definitiva del instrumento se empleó la técnica de conglomerados, seleccionando al azar centros educativos de cada una de las 6 áreas territoriales teniendo en cuenta dos criterios de inclusión: la necesaria participación de un centro rural por área territorial y mantener una proporción de un centro de régimen privado-concertado por cada tres de titularidad pública. En cada uno de los centros seleccionados se celebró una sesión única para la cumplimentación del cuestionario por parte del número de estudiantes necesarios para cubrir la cuota muestral definida. Antes de su aplicación, se solicitó permiso, tanto al Director General de Educación de cada Comunidad Autónoma, como los directores de los centros educativos seleccionados. Dos investigadores 
se encargaron personalmente de dirigir, ayudar y coordinar la cumplimentación del cuestionario en cada centro. De esta forma se garantizó la fiabilidad y se redujo la mortalidad experimental.

En este estudio se ha empleado, en dos fases, un análisis inferencial bivariado a través de tablas cruzadas y empleando los estadísticos V de Cramer y Phi, fijando como nivel de significatividad un valor de $p$ inferior a 0,05 . En ambos estadísticos se determinó que los valores absolutos menores o iguales a ,200 indicaban asociaciones muy débiles, entre ,200 y ,400 débiles, entre ,400 y ,600 moderadas, entre $, 600 \mathrm{y}, 800$ fuertes y muy fuertes para valores entre, 800 y 1 . En una primera fase se muestra el perfil de las familias que matriculan a sus hijos en centros educativos católicos de secundaria postobligatoria frente al perfil de las familias que optan por centros laicos. En una segunda fase, el estudio las tablas de contingencia profundizan en la comparación del perfil de las familias de centros educativos católicos que conviven con abuelos en el hogar con el de las familias de centros laicos.

\section{RESULTADOS}

Los datos de este estudio revelan que tan solo conviven tres generaciones en el mismo hogar (abuelos, padres e hijos) en el 7,3\% de las familias españolas con estudiantes de educación secundaria postobligatoria.

Profundizando en cómo son las familias de estos estudiantes se resalta que, en valores absolutos, en el $72,1 \%$ de estas familias los padres viven juntos, mientras que el $25,6 \%$ restante se reparte entre familias con padres separados $(19,4 \%)$, familias monoparentales $(6,2 \%)$ y entre familias con otras situaciones $(1,6 \%)$.

La distribución no equilibrada de los sectores poblacionales en función de la situación familiar obliga, para garantizar la consistencia del estudio, a operar con valores relativos. El estudio relacional destaca que la porción de familias en las que conviven las tres generaciones es similar en todos los sectores poblacionales definidos en función de la situación familiar ( $\mathrm{V}$ de $\mathrm{Cramer}=-, 083$ $p=, 063$ ) (Figura 1). 
Figura 1: Convivencia de tres generaciones y situación familiar.

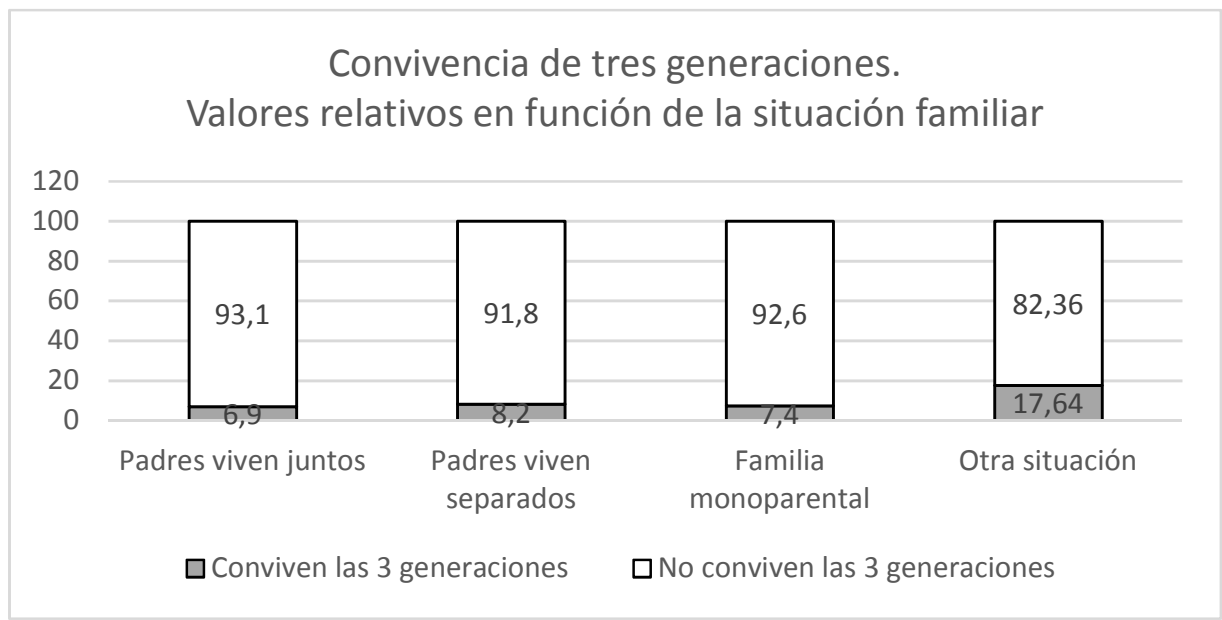

Centrando el análisis en la titularidad de los centros educativos cabe destacar que, en términos absolutos, los centros católicos reúnen al 17,29\% de los estudiantes de secundaria postobligatoria del estado español y al 13,79\% de las familias que comparten su hogar con los abuelos.

Al prestar atención a los valores relativos, se ratifica que en los centros católicos se encuentran menos familias en las que conviven tres generaciones (abuelos, padres e hijos) en el mismo hogar (V de Cramer $=, 026 p=, 018$ ). E1 8\% de las familias con hijos estudiando educación secundaria postobligatoria en centros laicos comparten hogar con los abuelos, frente al 6,2\% de las familias que se decantan por centros católicos (Tabla 2).

Tabla 2: Titularidad del centro de estudios y convivencia de tres generaciones en el hogar

\begin{tabular}{cccc}
\hline & $\begin{array}{c}\text { Centro educativo } \\
\text { católico }\end{array}$ & Centro educativo laico & Total \\
\hline $\begin{array}{c}\text { Viven sus } \\
\text { abuelos en el } \\
\text { domicilio } \\
\text { habitual }\end{array}$ & 16 & 100 & 116 \\
\hline $\begin{array}{c}\text { No viven sus } \\
\text { abuelos en el } \\
\text { domicilio } \\
\text { habitual }\end{array}$ & $6,2 \%$ & $8,0 \%$ & $7,7 \%$ \\
\hline Total & 242 & 1146 & 1388 \\
\hline
\end{tabular}

$P<, 005$ (260 datos perdidos) 
Asimismo, el estudio inferencial muestra cómo en los centros católicos se conservan más familias tradicionales que en los centros de otra titularidad (V de Cramer $=-, 084 p=, 042$ ). En este sentido, los padres del $84,5 \%$ de los estudiantes de centros católicos viven juntos, mientras que este porcentaje desciende hasta el $76,4 \%$ entre las familias con alumnos de Educación Secundaria Postobligatoria en centros laicos. Se destaca, en el sentido contrario, que los centros católicos se encuentran 1 y 6 puntos porcentuales por debajo de la media española en cuanto a familias monoparentales y con padres separados respectivamente (Tabla 3).

Tabla 3: Titularidad del centro de estudios y situación familiar

\begin{tabular}{cccc} 
& Centro educativo católico & Centro educativo laico & Total \\
& 218 & 943 & \\
\hline $\begin{array}{c}\text { Los padres } \\
\text { viven juntos }\end{array}$ & $84,5 \%$ & $76,4 \%$ & 1161 \\
\hline $\begin{array}{c}\text { Los padres } \\
\text { viven }\end{array}$ & 29 & 225 & $77,8 \%$ \\
separados & $11,2 \%$ & $18,2 \%$ & 254 \\
\hline $\begin{array}{c}\text { Solo tiene } \\
\text { madre o } \\
\text { padre }\end{array}$ & 9 & 56 & $17,0 \%$ \\
\hline $\begin{array}{c}\text { No tiene ni } \\
\text { padre ni } \\
\text { madre }\end{array}$ & $3,5 \%$ & $4,5 \%$ & 65 \\
\hline $\begin{array}{c}\text { Otra } \\
\text { situación } \\
\text { familiar }\end{array}$ & 0 & 2 & $4,4 \%$ \\
\hline Total & $0,0 \%$ & $0,2 \%$ & 2 \\
\hline & $2,8 \%$ & 8 & $0,1 \%$ \\
\hline
\end{tabular}

$P<, 005$ (272 datos perdidos)

En cuanto al nivel de estudios de los progenitores que comparten hogar con los abuelos, este estudio revela que, en valores absolutos, el $45,9 \%$ de los padres y el $39,2 \%$ de las madres no superan los estudios primarios, frente a un $20,5 \%$ y $26,4 \%$ respectivamente que declaran haber culminado estudios universitarios. No obstante, el análisis inferencial no muestra diferencias significativas a la hora de convivir abuelos, padres e hijos en un mismo hogar, ni en cuanto al nivel de 
estudios del padre (V de Cramer=-,063 $p=, 089$ ) ni al de la madre (V de Cramer $=, 038 p=, 480$ ). Esto refleja proporciones similares de familias en las que conviven tres generaciones en los distintos estratos educativos paternos y maternos (Figuras 2 y 3 ).

Figura 2: Convivencia de tres generaciones y nivel de estudios del padre

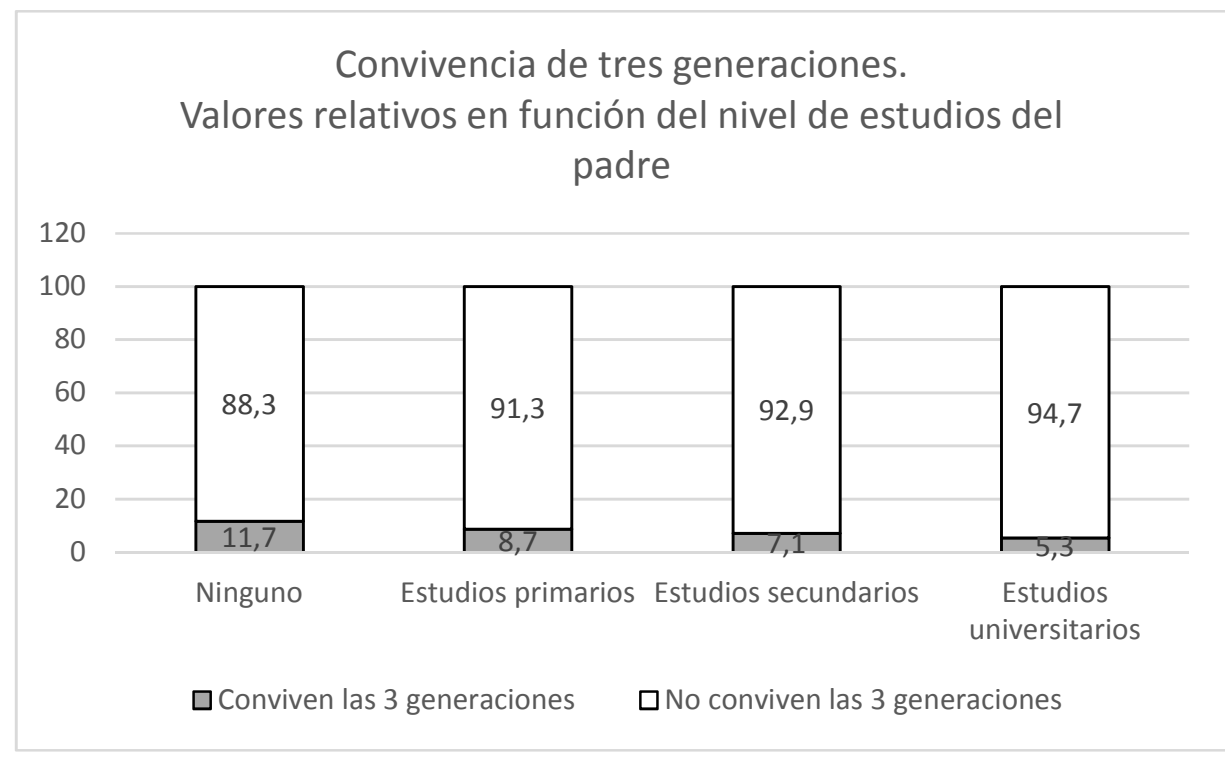

Figura 3. Convivencia de tres generaciones y nivel de estudios de la madre

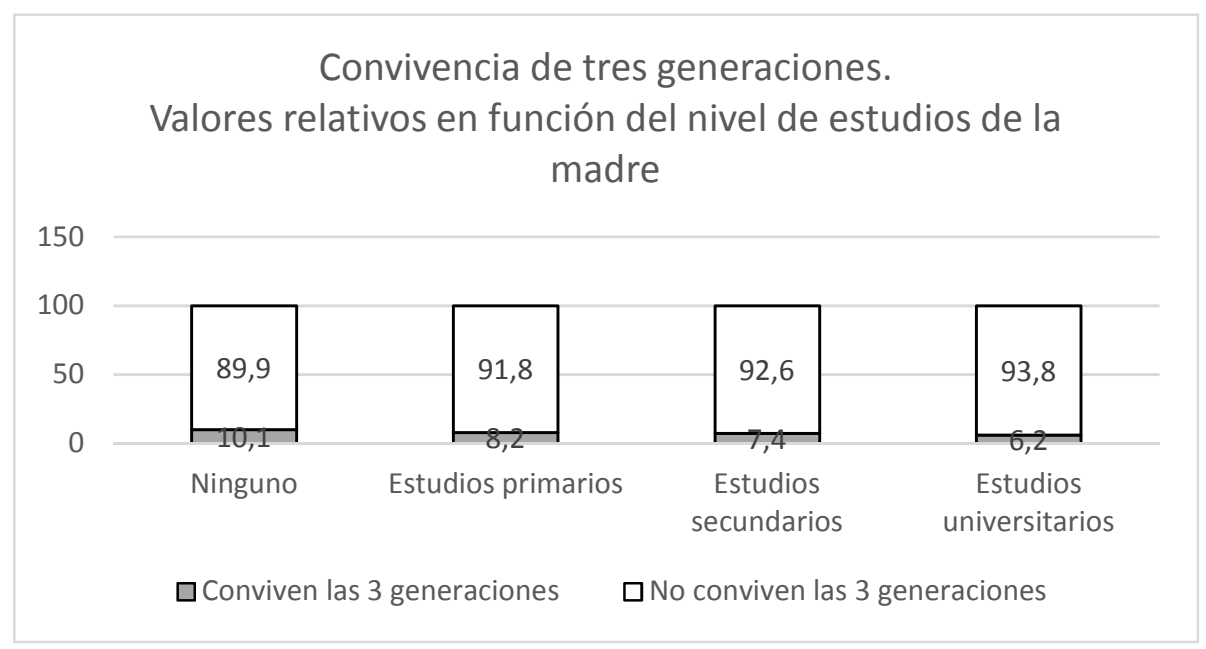


Sin embargo, sí se detectan diferencias significativas en cuanto al nivel de estudios del padre y el tipo de centro elegido para la formación postobligatoria de sus hijos. Los centros católicos cuentan con más padres sin estudios o con estudios universitarios que los centros laicos ( $\mathrm{V}$ de Cramer $=, 073 p=, 043$ ) (Tabla $5)$.

Tabla 5: Titularidad del centro de estudios y nivel de estudios del padre

\begin{tabular}{cccc}
\hline & $\begin{array}{c}\text { Centro educativo } \\
\text { católico }\end{array}$ & Centro educativo laico & Total \\
\hline Ningún & 16 & 46 & 62 \\
estudio & $6,3 \%$ & $3,9 \%$ & $4,3 \%$ \\
\hline Estudios & 66 & 383 & 449 \\
primarios & $26,2 \%$ & $32,6 \%$ & $31,4 \%$ \\
\hline Estudios & 84 & 409 & 493 \\
secundarios & $33,3 \%$ & $34,8 \%$ & $34,5 \%$ \\
\hline Estudios & 86 & 338 & 424 \\
universitarios & $34,1 \%$ & $28,7 \%$ & $29,7 \%$ \\
\hline \multirow{2}{*}{ Total } & 252 & 1176 & 1428 \\
& $100,0 \%$ & $100,0 \%$ & $100,0 \%$ \\
\hline
\end{tabular}

$P<, 005$ (336 datos perdidos)

No obstante, no se detectan diferencias significativas en cuanto al nivel de estudios de la madre (V de Cramer $=, 032 p=, 685$ ) (Tabla 6).

Tabla 6: Titularidad del centro de estudios y nivel de estudios de la madre

\begin{tabular}{cccc}
\hline & $\begin{array}{c}\text { Centro educativo } \\
\text { católico }\end{array}$ & Centro educativo laico & Total \\
\hline Ningún & 11 & 43 & 54 \\
estudio & $4,3 \%$ & $3,6 \%$ & $3,7 \%$ \\
\hline Estudios & 78 & 354 & 432 \\
primarios & $30,7 \%$ & $29,5 \%$ & $29,7 \%$ \\
\hline Estudios & 78 & 414 & 492 \\
secundarios & $30,7 \%$ & $34,4 \%$ & $33,8 \%$ \\
\hline Estudios & 87 & 391 & 478 \\
universitarios & $34,3 \%$ & $32,5 \%$ & $32,8 \%$ \\
\hline Total & 254 & 1202 & 1456 \\
& $100,0 \%$ & $100,0 \%$ & $100,0 \%$ \\
\hline
\end{tabular}

$p>, 005$ (308 datos perdidos) 
En esta línea, al segmentar los casos en función del tipo de centro educativo (católico/laico), tampoco se muestran diferencias significativas en cuanto al nivel de estudios del padre o de la madre de las familias en las que conviven tres generaciones.

En cuanto a la situación profesional de los padres, los datos absolutos indican que un $72,5 \%$ de los padres de las familias que conviven con sus abuelos se encuentran empleados, un $46,6 \%$ por cuenta ajena y un $25,9 \%$ por cuenta propia. En el lado opuesto, en situación de desempleo, se registran un 17,2\% de los padres. Tan solo un 4,3\% están jubilados y un escaso $1,7 \%$ se dedican a la atención del hogar. En el caso de las madres, la situación de empleo es alcanzada por una porción muy inferior a la de los padres $(57,5 \%)$, engordando en 23,3 unidades porcentuales el porcentaje de quienes se dedican al cuidado del hogar.

El análisis inferencial, en este caso, tampoco muestra diferencias significativas a la hora de compartir hogar abuelos, padres e hijos en función de la situación profesional ni del padre (V de Cramer $=, 077 p=, 099)$ ni de la madre ( $V$ de Cramer $=, 062 p=, 270$ ). Luego la porción de familias en las que conviven tres generaciones es similar en los distintos estratos profesionales paternos $y$ maternos (Figuras 4 y 5).

Figura 4: Convivencia de tres generaciones y situación profesional del padre

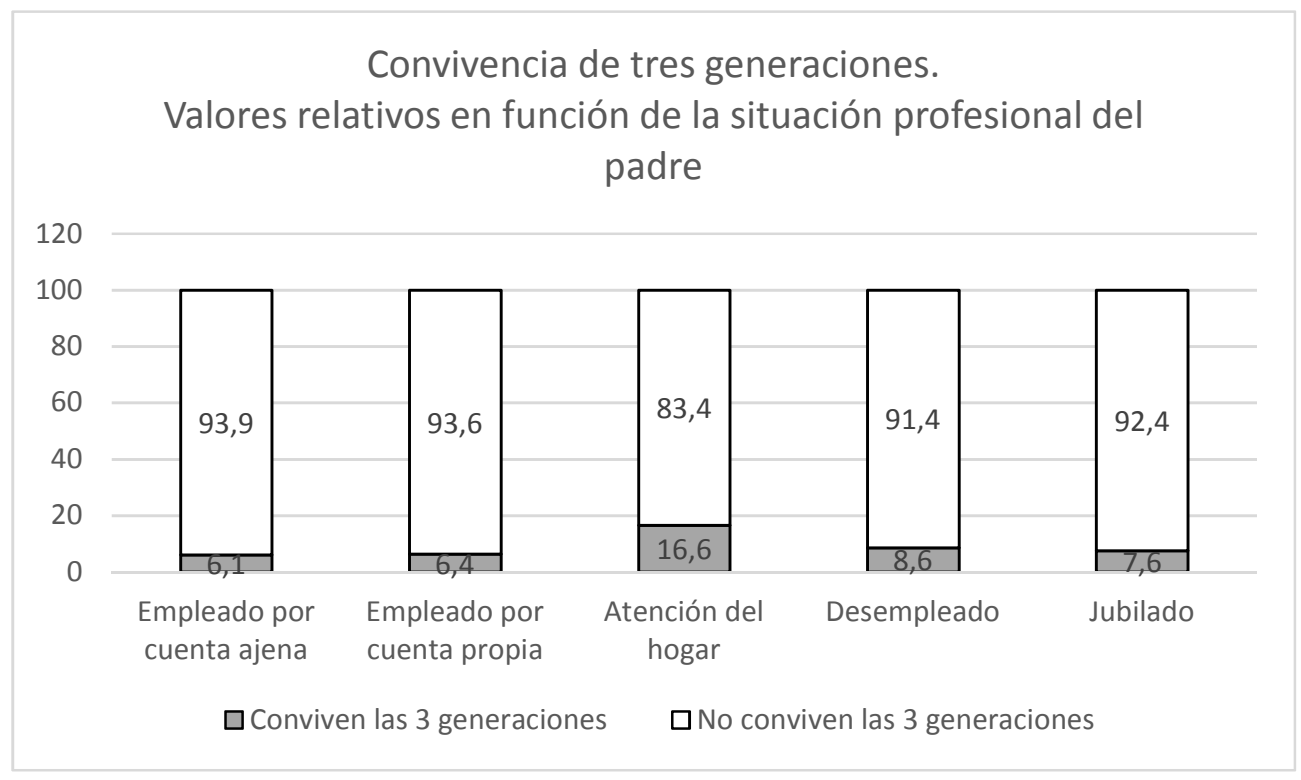


Figura 5: Convivencia de tres generaciones y situación profesional de la madre

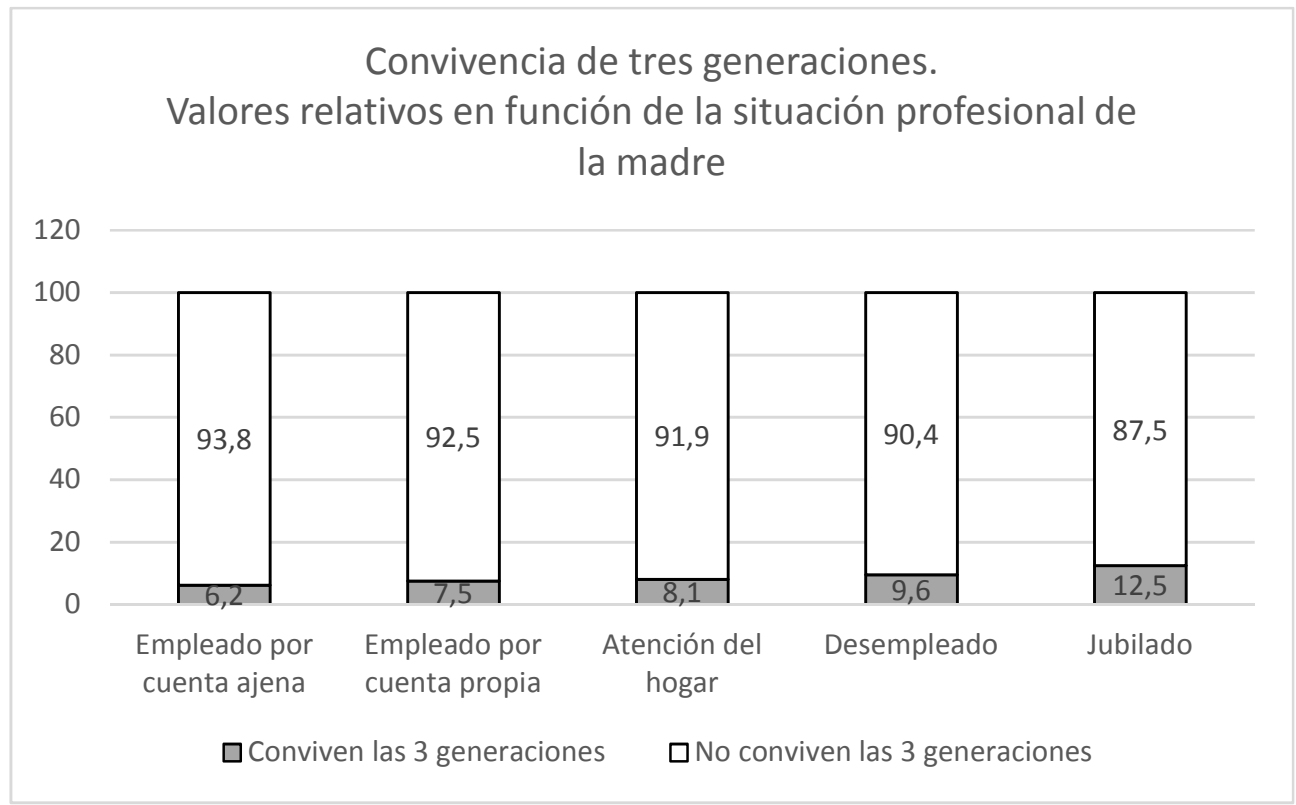

Tampoco se identifican diferencias significativas en cuanto a la situación profesional del padre ( $\mathrm{V}$ de Cramer $=, 069 p=, 250)$ ni de la madre ( $\mathrm{V}$ de Cramer $=, 065 p=, 305$ ) respecto al tipo de centro elegido para matricular a sus jóvenes.

En consecuencia, al segmentar los casos en función del tipo de centro educativo (católico/laico), tampoco se muestran diferencias significativas al estudiar la situación profesional del padre o de la madre de las familias que conviven con abuelos en el hogar.

\section{DISCUSIÓN}

Son pocas las familias españolas, cuyos hijos e hijas cursan Educación Secundaria Postobligatoria, las que están configuradas por tres generaciones, no estando esta condición vinculada a la situación familiar. Dicho de otro modo, la porción de familias trigeneracionales es similar tanto en aquellas en las que los progenitores viven juntos, como en las que éstos están separados, como en las monoparentales o las caracterizadas por otras situaciones diferentes a las señaladas. 
Los resultados obtenidos atestiguan que los centros de carácter religioso incluyen un mayor número de familias tradicionales que los centros de otra condición, lo que se sitúa en coherencia con la idiosincrasia que sustenta los proyectos educativos de estas instituciones. Si bien las escuelas católicas son, cada vez más, un reflejo de la sociedad actual, plural e integradora, que acoge a todo tipo de familias (Instituto Universitario de la Familia de la Universidad Pontificia de Comillas, 2016), el presente estudio constata que en los centros no religiosos es superior el número de familias monoparentales o aquéllas en las que los progenitores están separados.

Nuestros datos ponen de manifiesto que las familias que eligen los centros religiosos para escolarizar a sus hijos tienen mayor proporción de padres que poseen estudios universitarios, no desvelándose diferencias ni respecto al nivel de estudios de las madres ni sobre la situación profesional de ambos progenitores, cuando del tipo de centro elegido se trata. Estos datos se sitúan, parcialmente, en sintonía con Mancebón y Pérez (2007), quienes constataron un nivel de estudios más alto y una ocupación en profesiones que requieren mayor cualificación en las familias de los estudiantes matriculados en centros concertados que, como indica el Instituto Nacional de Estadística (2017) en el Informe de financiación y gastos de la enseñanza privada en España, ocupan alrededor del $55 \%$ de los centros religiosos. Asimismo, nuestra investigación destaca que los centros católicos cuentan con más padres sin estudios que las instituciones laicas, lo que puede ser debido a la mayor función asistencial que caracteriza los centros religiosos.

Focalizándonos en el objetivo que ocupa el presente estudio, puede afirmarse que es el tipo de familia, exclusivamente, un factor asociado al perfil de una familia en la que conviven tres generaciones, pues se descubre que cuando los progenitores están separados y sus hijos cursan estudios en centros laicos, es mayor el porcentaje de hogares en los que conviven las tres generaciones.

Con esta salvedad, y basándonos en los resultados obtenidos en esta investigación, puede afirmarse que no hay características diferenciadoras que predispongan a una configuración familiar formada por tres generaciones, dado que ni el nivel de estudios ni la situación profesional de los progenitores configuran este perfil familiar.

Esta realidad obliga a poner el foco de atención en las relaciones intergeneracionales, desde las políticas familiares, como un medio generador de procesos educativos y sociales (Fragüela, De-Juanas, y Franco, 2018), máxime cuando quedan constatadas las significativas implicaciones que tiene para sus 
integrantes esta relación, como es el caso de la influencia mutua en su desarrollo personal y social, la contribución de los mayores al equilibrio familiar o el refuerzo del vínculo emocional, además de la transmisión de habilidades útiles para la vida, de valores, de códigos morales, de normas sociales, así como de cultura e identidad (Álvarez y Rodríguez, 2008; Lloyd, 2008; Osuna, 2006).

En su conjunto, son conclusiones que avalan la necesidad de realizar un estudio de ámbito nacional que incluya variables como el nivel económico familiar, el estado de salud de los miembros de la unidad familiar, el estado civil de los abuelos o la tipología de la vivienda, con el fin de aportar argumentos a considerar en las políticas educativas, culturales y sociales. Ello facilitará criterios motivados para el diseño, la implementación y la evaluación de programas de acción-intervención-educación familiar, orientados a una doble línea de acción: por un lado, contrarrestando los factores que obstaculizan el desarrollo integral familiar en sus prácticas compartidas; y, por otro, acentuando aquellas realidades que contribuyen a mejorar su bienestar personal y comunitario, $\mathrm{y}$, por ende, su calidad de vida.

\section{REFERENCIAS BIBLIOGRÁFICAS}

Álvarez, J. y Rodríguez, C. (2008). El valor de la institución familiar en los jóvenes universitarios de la Universidad de Granada. Bordón, 60(1), 7-21.

Aranda, L. (2013). Doubling up: ¿a gift or a same? Multigenerational household and parental depression of older Europeans, 134, 12-22.doi: http://dx.doi.org/10.1016/j.socscimed.2015.03.056.

Badenes, N. y López, M.T. (2011). Doble dependencia: abuelos que cuidan nietos en España. Revista de servicios sociales, 49, 107-125. doi: http://dx.doi.org/ 10.5569/1134-7147.49.09

Bolívar, A. (2006). Familia y escuela: dos mundos llamados a trabajar en común. Revista de Educación, 339, 119-146.

Brazelton, T. y Greenspan, S. (2005). Las necesidades básicas de la infancia. Barcelona: Biblioteca de infantil.

Castillo, M. E. (2018). Prospectiva de la familia en la socialización de valores comunitarios. Revista Redine, 10(2), 56-59.

Cavalotti, R. (2015). La familia como comunidad de generaciones y la solidaridad familiar intergeneracional como su cohesión interna. Familia. Revista de Ciencias y Orientación Familiar, 51, 9-16. 
De-Juanas, A. y García-Castilla, F.J. (2018). Educación y ocio en jóvenes vulnerables (presentación). Pedagogía Social, Revista Interuniversitaria, 31, 13-17. doi: http://dx.doi.org/10.7179/PSRI_2018.31.01

De Fuenmayor, A., Granell, R., y Villareal, E. (2003). Determinantes de la elección de centro educativo por parte de los padres. Estudios de economía aplicada, 21(2), 377-389.

Demaray, M. K. \& Malecki, C. K. (2002). The relationship between perceived social support and maladjustment for student at risk. Psychology in the Schools, 39(3), 305-316. doi: http://dx.doi.org/10.1002/pits.10018

Elzo, J. (2004). Tipología y socialización de las familias españolas. Arbor, 702, 205-229. doi: http://dx.doi.org/10.3989/arbor.2004.i702.566

Encinas, M. R. (2007). Hombre, familia y amor: una visión evolucionista. Cauriensia, vol. II, 389-414.

Escardíbul, J. O. y Villarroya, A. (2009). Desigualdades en la elección de centro escolar en España. XVI Encuentro de Economía Pública. Granada.

Europapress (23 de julio de 2016). Los abuelos continúan siendo fundamentales en la estructura familiar española, sobre todo en materia de conciliación. Recuperado de http://www.europapress.es/epsocial/noticia-abuelos-continua n-siendo-fundamentales-estructura-familiar-espanola-todo-materiaconciliacion-20160723132956.html

Fragüela, R., De-Juanas, A. y Franco, R. (2018). Ocio deportivo en jóvenes potencialmente vulnerables: beneficios percibidos y organización de la práctica. Pedagogía Social, Revista Interuniversitaria, 31, 49-58. doi: http://dx.doi.org/10.7179/PSRI_2018.31.04

García-Roca, J. (1999). Composición y funciones básicas de la familia. En A. Gimeno, La familia: el desafío de la diversidad. Barcelona: Ariel.

González Bernal, J. y de la Fuente, R. (2007). Intergenerational Grandparent/ Grandchild Relations: The Socioeducational Role of Grandparents. Educational Gerontology, 34(1), 67-88. doi: http://dx.doi.org/10.1080/0360127070 1763993

Instituto Nacional de Estadística. (2017). Estadística de financiación y gastos de la Enseñanza privada. Madrid. Recuperado de https://www.ine.es/dyngs/INE base/es/operacion.htm?c=Estadistica_C\&cid $=1254736176760 \&$ menu $=$ result ados\&idp=1254735573113

Instituto Universitario de la Familia de la Universidad Pontificia Comillas (2016). Demandas educativas de los padres en España. Estudio de Escuelas Católicas. Madrid. 
Jackson, Y. \& Warren, J. S. (2000). Appraisal, social support, and life events: predicting outcomes behavior in schoolage children. Child Development, 71(5), 1441-1457. doi: http://dx.doi.org/10.1111/1467-8624.00238

Juang, L. P. \& Silbereisen, R. K. (1999). Supportive parenting and adolescent adjustment across time informer East and West German. Journal of Adolescence, 22(6), 719-736. doi: http://dx.doi.org/10.1006/jado.1999.0267

Lloyd, J. (2008). The State of Intergenerational Relations Today. London: ILCUK.

MacCallum, J., Palmer, D., Wright, P., Cumming-Potvin, W., Northcote, J., Booker, M., \& Tero, C. (2006). Communitybuildingthroughintergeneration alexchangeprograms. Australia: NationalYouthaffairsResearchScheme.

Mancebón, M. J. y Pérez, D. (2007). Conciertos educativos y selección académica y social del alumnado. Hacienda Pública Española, Revista de Economía Pública, 180, 77-106.

Marí-Klose, P. y Escapa, S. (2015). Solidaridad intergeneracional en época de crisis: ¿mito o realidad? Panorama Social, 22(2), 61-78.

Martínez de Miguel, S., Escarbajal, A., y Moreno, P. (2012). El rol de los abuelos en la relación con sus nietos. Una aproximación cualitativa desde el punto de vista socioeducativo. En G. Pérez (Coord.), Envejecimiento activo y solidaridad intergeneracional: claves para un envejecimiento activo (pp. 1-35). Madrid: UNED.

Megías, I. y Ballesteros, J. C. (2011). Abuelos y Abuelas...para todo: Percepciones en torno a la educación y el cuidado de los nietos. Madrid: FAD.

Organización Mundial de la Salud (2015). Informe Mundial sobre el envejecimiento y la salud. Recuperado de http://apps.who.int/iris/bitstream/10665/18 6466/1/9789240694873_spa.pdf.

Osuna, M. J. (2006). Relaciones familiares en la vejez: vínculos de los abuelos y de las abuelas con sus nietos y nietas en la infancia. Revista Multidisciplinar de Gerontología, 16(1), 16-25.

Peña Acuña, B. y Pezzoli, S. (2018). Propuesta de comunicación intergeneracional entre tercera edad y jóvenes en la sociedad del conocimiento. Revista de Comunicación de la SEECI, 45, 01-14. doi: http://doi.org/10.15198/seeci. 2018.45.01-14.

Romero, C. (2002). La familia desde una perspectiva sistémico-dinámica. En: Gervilla, E. (Coord.), Educación familiar. Nuevas relaciones humanas y humanizadoras (pp. 155-165). Madrid: Narcea. 
Sellés, J. F. (2006). La filiación personal humana. Estudio acerca de su lo más radical de la antropología es ser hijo. Cauriensia, 1, 201-217.

Valdemoros, M. A., Ponce de León, A., Sanz, E., y Caride, J. A. (2014). La influencia de la familia en el ocio físico-deportivo juvenil: nuevas perspectivas para la reflexión y la acción. Arbor, 190 (770): a192. doi: http://dx.doi.org/10.3989/arbor.2014.770n6013

Magdalena Saenz de Jubera Ocón Facultad de Letras y de la Educación Universidad de La Rioja Universidad de La Rioja, Luis de Ulloa 2, 26004 Logroño (España) https://orcid.org/ 0000-0001-8086-907

\section{Rosa Ana Alonso Ruiz} Facultad de Letras y de la Educación Universidad de La Rioja Universidad de La Rioja, Luis de Ulloa 2, 26004 Logroño (España) https://orcid.org/0000-0003-3215-578
Ana Ponce de León Elizondo Facultad de Letras y de la Educación Universidad de La Rioja Universidad de La Rioja, Luis de Ulloa 2, 26004 Logroño (España) https://orcid.org/0000-0003-4622-8062

Ángel De-Juanas Oliva Facultad de Educación UNED Juan del Rosal, 14 28040 Madrid (España) https://orcid.org/0000-0003-0103-7860 
